\title{
Representation of the Axial Settings of Mica Polytypes
}

\author{
Massimo Nespolo, ${ }^{a} *$ Hiroshi Takeda ${ }^{b}$ and Giovanni Ferraris ${ }^{c}$ \\ ${ }^{a}$ Mineralogical Institute, Graduate School of Science, University of Tokyo, 7-3-1 Hongo, Bunkyo-ku, Tokyo 113- \\ 0033, Japan, ${ }^{b}$ Research Institute, Chiba Institute of Technology 2-17-1 Tsudanuma, Narashino, Chiba 275-0016, \\ Japan, and ${ }^{c}$ Department of Mineralogical and Petrologic Sciences, University of Torino, via Valperga Caluso, 35, \\ 10125 Torino, Italy. E-mail: nespolo@min.s.u-tokyo.ac.jp
}

(Received 14 July 1997; accepted 12 December 1997)

\begin{abstract}
The basic unit of mica polytypes has monoclinic symmetry and the layer stagger is a submultiple of the periodicity along the $a$ axis. Because of these features, more than one suitable axial setting can be chosen for non-orthogonal micas. Three types of axial settings are introduced and shown to be useful for classifying nonorthogonal polytypes of micas and indexing their diffraction patterns. Standard setting is the axial setting of a polytype leading to the shortest projection of the $c$ axis onto the (001) plane. Basic axial setting is the standard setting of a polytype with a number $N$ of layers equal to an integral multiple of $3^{n}$. All the polytypes having the same basic axial setting belong to the same Series. Fixed-angle setting is the axial setting of a general polytype showing the same angle as the corresponding basic axial setting. The total layer stagger of stacking classifies polytypes into two Classes: their $c$ axis is inclined towards respectively the shortest (Class $a$ ) or the longest (Class $b$ ) of the two orthohexagonal axes in the plane of the layer. Each Class is further divided according to $N=1(\bmod 3)($ Subclass 1$)$ and $N=2(\bmod 3)$ (Subclass 2$)$. By expressing $N$ as $3^{n}(3 K+L)$, the two integers $n$ and $L(1$ or 2$)$ establish the Series and the Subclass, respectively. This definition allows an effective classification of the polytypes and a systematic approach to the indexing of diffraction patterns, independently of their complexity, which increases with $N$. The transformation rules between settings are given and examples are discussed.
\end{abstract}

\section{Introduction}

Approaches using numeric representations (Ross et al., 1966; Takeda \& Sadanaga, 1969; Zvyagin, 1962, 1967, 1974; Zvyagin et al., 1979; Dornberger-Schiff, Backhaus \& Durovič, 1982; Dornberger-Schiff, Ďurovič \& Zvyagin, 1982; Backhaus \& Durovič, 1984; Ďurovič et al., 1984; Weiss \& Wiewióra, 1986; Zhukhlistov et al., 1990; Takeda \& Ross, 1995), vector schemes (Smith \& Yoder, 1956; Takéuchi \& Haga, 1971) or both (Dekeyser \& Amelinckx, 1953; Thompson, 1981) have been introduced to describe mica polytypes, whose

(C) 1998 International Union of Crystallography

Printed in Great Britain - all rights reserved features derive from the stacking in a complex way of a simple unit layer (Hendricks \& Jefferson, 1939). Mica polytypes exhibit local symmetry higher than that shown by their space group and unit-cell translations (Sadanaga \& Takeda, 1968).

The choice of the axial setting represents an important step in comparing calculated and experimental patterns, especially when the number of layers increases. Different axial settings have been used in the literature; in some cases, the projection of the $c$ axis onto the (001) plane was kept constant, in others, it was the monoclinic angle, and in others neither was kept. Furthermore, examples can be traced where a mix of different axial settings is used to index different reciprocal-lattice planes of the same polytype or where the setting of a polytype is used to index the pattern of a different polytype (Ross et al., 1966; polytypes $3 T, 3 A_{1}$, $\left.4 M_{1}, 4 M_{2}, 4 M_{3}, 4 A_{8}\right)$. $\dagger$ In the present work, the axial settings for mica polytypes are for the first time defined in a systematic way and the transformation rules between them are given. This is the first treatment leading to a completely general approach to the problem of the choice of mica axial settings. It might also be used as the starting point for similar generalizations in cases of other phyllosilicates, which share many geometrical features with micas.

In the following, we shall constantly refer to the space-fixed orthohexagonal reference introduced by Zvyagin (1962). However, different structure-related axial settings will be introduced in both direct and reciprocal space. The relation with Zvyagin's setting will be kept by means of the transformation matrices, which will be given according to Hahn (1983) and Arnold (1983): covariant and contravariant quantities written as row and column matrices, respectively. According to Hahn (1983), bold letters (e.g. a, b, c) indicate vectors, while their lengths are written as standard italic letters (e.g. $a, b, c)$; axes are also written as standard italic letters.

† According to the suggestions of the IUCr Ad Hoc Committee on the Nomenclature of Polytypes (Guinier et al., 1984), the lattice symbol for triclinic polytypes should be changed from $T c$ to $A$ (anorthic). The polytype called $4 A_{1}$ in Ross et al. (1966) has been called $4 A_{8}$ in Takeda \& Ross (1995) and vice versa. 
Table 1. Metrical relations in orthohexagonal and monoclinic settings

Orthohexagonal setting

$$
b=a \times 3^{1 / 2} \quad\left\{\begin{array}{l}
a^{*}=1 / a \\
b^{*}=1 / b \\
c^{*}=1 / c
\end{array}\right\} \quad b^{*}=a^{*} / 3^{1 / 2}
$$$$
b=a \times 3^{1 / 2}
$$

\section{Unit layer}

The ideal mica unit layer is built up by sandwiching an octahedral $(O)$ sheet with symmetry $P(\overline{3}) 1 m$ [notation after Dornberger-Schiff (1959); details in Merlino (1990)] between two tetrahedral $(T)$ sheets with symmetry $P(6) \mathrm{mm}$, which is reduced to $P(3) 1 \mathrm{~m}$ by the so-called ditrigonal rotation within the tetrahedral sheet (Radoslovich, 1959; Radoslovich \& Norrish, 1962; Takeda \& Sadanaga, 1969). The two $T$ sheets show a relative shift of $a / 3$ so that the resulting layer symmetry is finally $C 12 / m(1)$ (Pabst, 1955). Rare cases of noncentrosymmetric layers (e.g. Brown, 1978) are neglected in this paper. The symmetry of the single layer structure ( $1 M$ polytype) is $C 2 / m, b$-unique setting. Two adjacent $T$ sheets belonging to different layers are always exactly facing each other in the ideal structure $[P(6) \mathrm{mm}$ symmetry of the tetrahedral sheets].

Neglecting small deviations from ideality, all mica polytypes are based on a hexagonal plane lattice [(001) plane], while in three dimensions they are metrically at least monoclinic. $\dagger$ The ideal mica lattice can thus be monoclinic, orthorhombic or trigonal and hexagonal: in the plane of the layer, two hexagonal axes $a_{1}$ and $a_{2}$ can be chosen but the axial setting based on them is useful only in dealing with trigonal and hexagonal polytypes. A $C$-centred unit cell, based on orthohexagonal $a$ and $b$ axes (orthohexagonal metric: $b=a \times 3^{1 / 2}, \gamma=\pi / 2$ ) and with the shortest $c$ axis, displays the complete symmetry of the layer. This is the cell most commonly used to describe the $1 M$ polytype and it is called the conventional cell according to Burzlaff et al. (1983). The reduction algorithm to the reduced cell is well known. Here the orthohexagonal $a$ and $b$ axes, corresponding to the cell called $C_{1}$ in Arnold (1983), is adopted. Finally, for all mica polytypes, a multiple (ideally) orthohexagonal cell can always be chosen whose axes will be kept coincident with those of Zvyagin's spacefixed reference. For non-orthogonal $N$-layer polytypes, it can be obtained by stacking three $N$-layer repeats. The metrical relations in direct and reciprocal space, in both the orthohexagonal and monoclinic settings, are given in Table 1. If $c^{\prime}$ is the vertical axis of the orthohexagonal sextuple cell and $c$ that of the monoclinic cell, keeping unchanged the orientation of the axes in

$\dagger$ The mica real-plane lattice is pseudohexagonal. The effect of the deviation from ideal geometry in phyllosilicates has been studied in detail for kaolin minerals (Zvyagin \& Drits, 1996) and seems to influence the formation of different polytypes. the plane of the layer, the transformation between monoclinic and orthogonal $C_{1}$ setting is as follows:

$$
\begin{aligned}
\left(\begin{array}{lll}
a & b & c^{\prime}
\end{array}\right) \Pi & =\left(\begin{array}{lll}
a & b & c
\end{array}\right), \\
\Pi & =\left[\begin{array}{lll}
1 & 0 & \frac{1}{3} \\
0 & 1 & 0 \\
0 & 0 & \frac{1}{3}
\end{array}\right], \quad V=V^{\prime} / 3, \\
\left(\begin{array}{lll}
a & b & c
\end{array}\right) \Pi^{-1} & =\left(\begin{array}{lll}
a & b & c^{\prime}
\end{array}\right), \\
\Pi^{-1} & =\left[\begin{array}{lll}
1 & 0 & 1 \\
0 & 1 & 0 \\
0 & 0 & 3
\end{array}\right], \quad V^{\prime}=3 V .
\end{aligned}
$$

\section{Classification of polytypes}

An $N$-layer mica polytype is built by stacking $N$ layers with a rotation between successive layers that is an integral multiple of $60^{\circ}$ (Smith \& Yoder, 1956). Geometrical features of mica polytypes can be simply expressed with the aid of two parameters (notation from Zvyagin, 1997): $\mathbf{c}_{n}$, which is the projection of the $c$ axis on (001), and $\mathbf{c}_{o}$, which is the projection of the $c$ axis on the $c^{*}$ axis. The projection $\mathbf{c}_{n}$, after subtraction of integers, can always be reduced to one of three possible values $(0,0),(\overline{1} / 3,0)$ or $(0, \overline{1} / 3)$ (Zvyagin, 1967). In the first case, a metrically orthogonal polytype is obtained with $\mathbf{c} \equiv \mathbf{c}_{o}$; the smallest cell based on the orthohexagonal axes coincides with the orthohexagonal sextuple cell introduced above and is thus a double $C$-centred cell. For trigonal and hexagonal polytypes, the choice of a primitive hexagonal cell is always possible. The other two cases correspond to nonorthogonal metrically monoclinic polytypes, which can be classified as follows: (i) polytypes with the $c$ axis inclined towards the $a$ axis of the space-fixed reference: these polytypes are grouped under the name Class a (b-unique setting); (ii) polytypes with the $c$ axis inclined towards the $b$ axis of the space-fixed reference: these polytypes are grouped under the name Class $b$ (a-unique setting). This classification is not simply a matter of names but corresponds to different values of the projection $\mathbf{c}_{n}$, which is a geometrical feature of nonorthogonal polytypes. The proposed two Classes replace the previous definition of $1 M$ type and $2 M_{2}$ type (Takeda \& Sadanaga, 1969), which is too restrictive, 
Table 2. Metrical relations in direct and reciprocal space for both Classes

$\begin{array}{lcc} & \text { Class } a & \text { Class } b \\ \text { Direct space } & c \cos \beta=-a / 3 & c \cos \alpha=-b / 3 \\ \text { Reciprocal space } & a^{*} \cos \beta^{*}=c^{*} / 3 & b^{*} \cos \alpha^{*}=c^{*} / 3\end{array}$

because it includes also the definition of the monoclinic angle for all polytypes with $N \neq 3 K$ ( $K \geq 0$, integer). The metrical relations characterizing these two Classes, with respect to the space-fixed reference, are given in Table 2. For polytypes with $N$ and $N^{\prime}$ layers, the following formulae hold (Zvyagin, 1967):

$$
\begin{array}{cc}
\text { Class } a & \text { Class } b \\
\tan \beta_{N}=\left(N / N^{\prime}\right) \tan \beta_{N^{\prime}} & \tan \alpha_{N}=\left(N / N^{\prime}\right) \tan \alpha_{N^{\prime}} .
\end{array}
$$

In a monoclinic metric, analogous relations hold also for the corresponding angles in the reciprocal space.

As shown by Takeda \& Sadanaga (1969), when $N=3 K$ the choice of the axial setting is geometrically limited. For a better understanding of these limitations, it is useful to express the number of layers contained in a repeat unit of each polytype in terms of the powers of 3. The set of positive integers as a function of the powers of 3 assumes the expression:

$$
N=3^{n}(3 K+L) \quad(n \geq 0, L=1 \text { or } 2) .
$$

In (3), three characteristic integers are used: $n, L$ and $K$. It will be shown that $n$ characterizes polytypes that can be based on an axial setting with constant monoclinic angle, whereas $L$ characterizes the transformation rule between different axial settings. Therefore, the following definitions are introduced: Series, which corresponds to the number $n$; and Subclass, which corresponds to the number $L$. The two Subclasses 1 and 2 are correspondingly defined. For each Series, $K=0$ of Subclass 1 determines the axial setting of the first polytype of the Series. This axial setting is called the basic axial setting and on it, for each polytype of the Series, an axial setting always having the same monoclinic angle can be built.

\section{Axial settings}

The basal nodes of the $C$ lattice are described by the vector $(p / 2, q / 2)$, where $p$ and $q$ are coprime integers of the same parity. In the case of non-orthogonal mica polytypes, $\mathbf{c}_{n}=a / 3$ or $b / 3$. Therefore, the $\mathbf{c}_{n}^{\prime \prime}$ projection of any $\mathbf{c}^{\prime \prime}$ vector defining $C$ cells of constant volume for those polytypes is obtained adding $(p / 2, q / 2)$ to $(\overline{1} / 3,0)$ or $(0, \overline{1} / 3)$. Using the metrical relation between $a$ and $b$ axes $\left(b=a \times 3^{1 / 2}\right)$, the modulus of $\mathbf{c}_{n}^{\prime \prime}$ is obtained as follows:

$$
\begin{aligned}
& c_{n}^{\prime \prime} / a=\left[p^{2} / 4+3 q^{2} / 4-(3 p-1) / 9\right]^{1 / 2} \\
& c_{n}^{\prime \prime} / b=\left[p^{2} / 12+q^{2} / 4-(3 q-1) / 9\right]^{1 / 2}
\end{aligned}
$$

for Class $a$ and Class $b$ polytypes, respectively.

\subsection{Class a}

The monoclinic axial setting of Class $a$ having $c_{n}=a / 3$ corresponds to the conventional cell: it will be called the Standard setting $\left({ }^{a} \mathbf{S}\right)$. The alternative setting will be any other possible $C$-centred setting. Labelling ${ }^{a} \mathbf{S}_{N}$ the standard setting common to all the polytypes with the same number of layers, the basic axial setting for all Class $a$ polytypes with $N \neq 0(\bmod 3)$ is labelled ${ }^{a} \mathbf{S}_{1}$.

\subsection{Class $b$}

In the case of Class $b$ polytypes, the definition of axial settings is a little different. In (1), matrices $\Pi$ and $\Pi^{-1}$ have the (13) and (23) elements exchanged because the $c$ axis is inclined towards $b$ and not $a$. The corresponding transformation:

$$
\begin{aligned}
\left(\begin{array}{lll}
a & b & c^{\prime}
\end{array}\right) \Pi & =\left(\begin{array}{lll}
a & b & c
\end{array}\right), \\
\Pi & =\left[\begin{array}{lll}
1 & 0 & 0 \\
0 & 1 & \frac{1}{3} \\
0 & 0 & \frac{1}{3}
\end{array}\right], \quad V=V^{\prime} / 3, \\
\left(\begin{array}{lll}
a & b & c
\end{array}\right) \Pi^{-1} & =\left(\begin{array}{lll}
a & b & c^{\prime}
\end{array}\right), \\
\Pi^{-1} & =\left[\begin{array}{lll}
1 & 0 & 0 \\
0 & 1 & 1 \\
0 & 0 & 3
\end{array}\right], \quad V^{\prime}=3 V
\end{aligned}
$$

leads to an $a$-unique monoclinic setting. It is common practice to exchange $a$ and $b$ axes (Smith \& Yoder, $1956)$, reverting to a $b$-unique monoclinic setting. The $a$-unique setting will be called Class $b$ Transitional setting $\left({ }^{b} \mathbf{T}\right)$ and, by analogy with Class $a$, the Standard setting $\left({ }^{b} \mathbf{S}\right)$ will be that deriving from the axial exchange. The ${ }^{b} \mathbf{T} \rightarrow{ }^{b} \mathbf{S}$ transformation involves the exchanges $a \rightarrow-b, b \rightarrow-a$, and $c \rightarrow-c$ in order to resume to a right-handed setting with non-acute $\beta$ angle, so that this axial transformation is accomplished by the matrix

$$
\nabla=\left[\begin{array}{ccc}
0 & \overline{1} & 0 \\
\overline{1} & 0 & 0 \\
0 & 0 & \overline{1}
\end{array}\right]_{b \mathbf{T} \rightarrow b \mathbf{S}},
$$

which is an orthogonal matrix, i.e. a real and symmetric matrix whose inverse coincides with the matrix itself: $\nabla=\nabla^{-1}$. All the other possible settings with the same $(\mathbf{a}, \mathbf{b})$ basis, are grouped under the definition of alternative settings. 
In the case of Class $b$ polytypes, there is no 1-layer polytype and the basic axial setting is defined by analogy with Class $a$ (Fig. 1). Having already defined ${ }^{a} \mathbf{S}_{1}$ setting, its relation to ${ }^{a} \mathbf{S}_{2}$ can be derived taking into account that $c_{n}(2)=c_{n}(1)$ and $c_{o}(2)=2 c_{o}(1)$ :

$$
\begin{aligned}
&\left(\begin{array}{lll}
a & b & c
\end{array}\right)_{a \mathbf{s}_{1}}=\left(\begin{array}{lll}
a & b & c
\end{array}\right)_{a \mathbf{s}_{2}}\left[\begin{array}{lll}
\overline{1} & 0 & \frac{1}{2} \\
0 & \overline{1} & 0 \\
0 & 0 & \frac{1}{2}
\end{array}\right] \\
&\left(\begin{array}{l}
a^{*} \\
b^{*} \\
c^{*}
\end{array}\right)_{a \mathbf{s}_{1}}=\left[\begin{array}{lll}
\overline{1} & 0 & 1 \\
0 & \overline{1} & 0 \\
0 & 0 & 2
\end{array}\right]\left(\begin{array}{l}
a^{*} \\
b^{*} \\
c^{*}
\end{array}\right)_{a \mathbf{s}_{2}},
\end{aligned}
$$

i.e. the Standard setting of the simplest polytype belonging to this Class ( $2 M_{2}$ polytype) is obtained from the $C_{1}$ setting through matrices $\Pi$ and $\nabla$, defined in $(1 a)$ and (5). The basic axial setting for Class $b$ (labelled ${ }^{b} \mathbf{S}_{1}$ ) is obtained by changing the superscript $a$ into $b$ in (6). The axis $a^{*}$ of ${ }^{b} \mathbf{S}_{1}$ contains the $\overline{2} 01$ reciprocal-lattice point (indexing in ${ }^{b} \mathbf{S}_{2}$ ). The geometrical definition of the basic axial settings for the two Classes is exactly the same. Taking into account that $c_{o}$ is the same both in ${ }^{b} \mathbf{S}_{1}$ and in ${ }^{a} \mathbf{S}_{1}$, while $c_{n}$ is $-b / 3$ in ${ }^{b} \mathbf{S}_{1}$ but $-a / 3$ in ${ }^{a} \mathbf{S}_{1}$, the value of the monoclinic angle of ${ }^{b} \mathbf{S}_{1}$ is approximately $107^{\circ}$.

\subsection{Fixed-angle setting for Class a}

The particular alternative setting based on ${ }^{a} \mathbf{S}_{1}$, i.e. having the same monoclinic angle but $c(N)=N c(1)$, is defined as the Fixed-angle setting $\left({ }^{a} \mathbf{F}_{N}\right)$. For a $1 M$ polytype: ${ }^{a} \mathbf{S}_{1} \equiv{ }^{a} \mathbf{F}_{1}$. In Fig. 2, the $(h 0 l)$ plane of ${ }^{a} \mathbf{S}$ is shown. The actual values of $a^{*}, c^{*}$ and $\beta^{*}$ depend on the polytype.

Remembering the metrical relations for Class $a$ in reciprocal space (Table 2), the projections on the $c^{*}$ axis

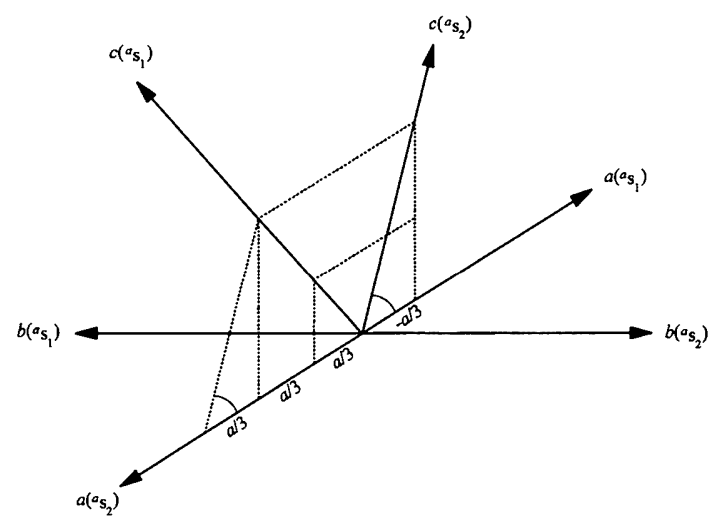

Fig. 1. Geometrical definition of basic axial setting for Class a polytypes starting from the Standard setting of the 2-layer polytype $\left(2 M_{1}\right)$. By exchanging $a$ and $b$ axes, the same procedure converts the Standard setting of the $2 M_{2}$ polytype into the basic axial setting for Class $b$ polytypes. of the vectors $\mathbf{r}^{*}=h \mathbf{a}^{*}+k \mathbf{b}^{*}+l \mathbf{c}^{*}$ are $(3 l \pm 2) c^{*} / 3$ so that an integral multiple of $c^{*}$ is never obtained. The $a^{*}$ axis of the Fixed-angle setting passes on $\pm 20 l\left({ }^{a} S\right.$ indexing), where the value of $l$ depends on $N$. Labelling ${ }^{o} \beta_{N}^{*}$ the angle between the $c^{*}$ and $a^{*}$ axes of the Fixedangle setting of an $N$-layer polytype, the following relationship holds:

$$
\tan \beta_{N}^{*}(h= \pm 2)=[(3 l \pm 2) / 2] \tan ^{o} \beta_{N}^{*} .
$$

Now, putting $N^{\prime}=1$ in the reciprocal-space equation corresponding to (2) for Class a polytypes, since by definition $\beta_{1}^{*}={ }^{o} \beta_{1}^{*}={ }^{o} \beta_{N}^{*}$, gives

$$
\tan ^{\circ} \beta_{N}^{*}=(1 / N) \tan \beta_{N}^{*} \text {. }
$$

Comparison of (8) and (7) gives $N=(3 l \pm 2) / 2$. The possible solutions are those leading to integral values of l, i.e:

$$
\begin{aligned}
& h=\overline{2}: l=2 K+2 \quad(\text { for } N=3 K+2) \\
& h=2: l=2 K \quad(\text { for } N=3 K+1) .
\end{aligned}
$$

No solution can be found for $N=3 K$. Therefore, if and only if $N \neq 3 K$, the ${ }^{a} \mathbf{F}$ setting can be used. The general transformation rule between ${ }^{a} \mathbf{S}$ and ${ }^{a} \mathbf{F}$ settings can be obtained by requiring that $a^{*}$ of ${ }^{a} \mathbf{F}$ contains the node $h 0 l$ with

$$
h=2(-1)^{L-1} ; \quad l=2(K+L-1) .
$$

For polytypes belonging to Subclass 1 or 2 , the $+a^{*}$ semi-axis of ${ }^{a} \mathbf{F}$ is respectively in the same or the opposite half-plane containing the $+a^{*}$ semi-axis of the ${ }^{a} \mathbf{S}$. It follows that for Subclass 2 polytypes ${ }^{a} \mathbf{S}_{N}$ is obtained from the basic axial setting $\left({ }^{a} \mathbf{S}_{1}\right.$, which coincides with ${ }^{a} \mathbf{F}_{1}$ and is the basis for obtaining $\left.{ }^{a} \mathbf{F}_{N}\right)$, changing sign to $a$ and $b$ and taking $c(N)=$ $(3 K+2) c_{1}+(K+1) a$. For Subclass 1, instead, $a$ and $b$

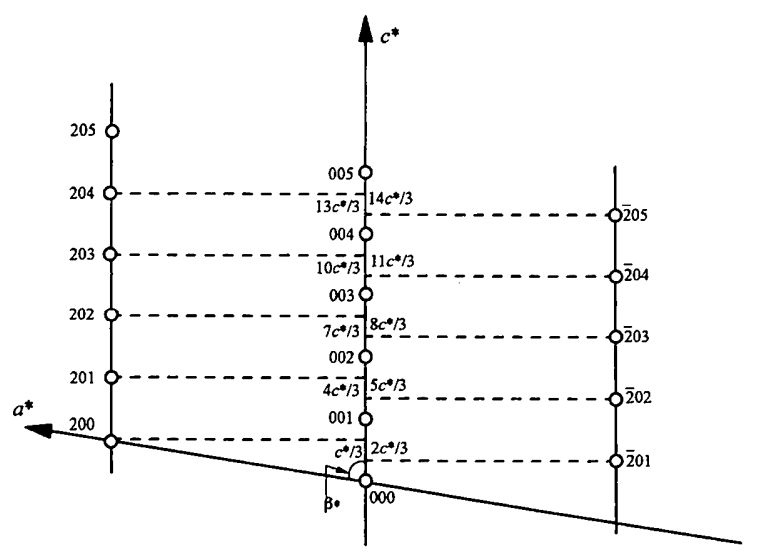

Fig. 2. Definition of Fixed-angle setting for multilayer polytypes. The ( $h 0 l$ ) plane of the ${ }^{a} \mathbf{S}$ setting is shown, limited to $h=0, \pm 2$ and $l$ from 0 to 5 . The projection of reciprocal-lattice nodes on the $c^{*}$ axis never corresponds to an integral multiple of the period along $c^{*}$. 
remain unchanged and $c(N)=(3 K+1) c_{1}+K a$. In this way, we resume a $c_{n}$ projection of $-a / 3(a / 3$ along $+a$ of ${ }^{a} \mathbf{S}_{1}$ setting for Subclass 2, and along $-a$ of the same setting for Subclass 1). The ${ }^{a} \mathbf{F}$ setting is based on ${ }^{a} \mathbf{S}_{1}$ but its $c_{o}$ is the same as in ${ }^{a} \mathbf{S}_{N}$ setting. Therefore, the matrix transforming the ${ }^{a} \mathbf{S}$ into ${ }^{a} \mathbf{F}$ does not depend on $N$. The general expression for the transformation matrices of both Subclasses, as a function of $K$ and $L$, is

$$
\Xi_{L}=\left[\begin{array}{ccc}
(-1)^{L-1} & 0 & (-1)^{L}(K+L-1) \\
0 & (-1)^{L-1} & 0 \\
0 & 0 & 1
\end{array}\right]
$$

It should be noted that $\Xi_{1} \neq \Xi_{1}^{-1}$ and $\Xi_{2}=\Xi_{2}^{-1}$. The matrices accomplishing the transformations from the orthohexagonal space fixed reference are $\Gamma_{L}=\Pi \Xi_{L}$.

\subsection{Fixed-angle setting for Class $b$}

The Fixed-angle setting for this Class, ${ }^{b} \mathbf{F}$, is built on ${ }^{b} \mathbf{S}_{1}$ by taking $N$ times the period along $c$. As for Class $a$, ${ }^{b} \mathbf{F}$ is not possible for polytypes with a number of layers $N$ that is an integral multiple of three. Starting from ${ }^{b} \mathbf{S}$, the general features of Class $b$ polytypes are the same as those of Class $a$. In fact, Fig. 2 and equations (7)-(10) apply also to Class $b$ if $a^{*}<b^{*}$ is assumed. Furthermore, since the geometrical definition of the basic axial setting is the same for both Classes, following the above procedure, identical transformation matrices are obtained. However, the following two facts should be kept in mind: (i) ${ }^{a} \mathbf{S}$ always has $a$ and $b$ axes coincident with those of the space-fixed setting [equation (1)]; (ii) in Class $b$, the setting having $a$ and $b$ coincident with those of the space-fixed setting, is the $a$-unique setting ${ }^{b} \mathbf{T}$, while the $b$-unique ${ }^{b} \mathbf{S}$ setting is obtained from ${ }^{b} \mathbf{T}$ through application of the $\nabla$ matrix. The matrix accomplishing the direct transformation from ${ }^{b} \mathbf{T}$ to ${ }^{b} \mathbf{F}$ is

$$
\begin{aligned}
{ }^{\nabla} \Xi_{L} & =\nabla \Xi_{L} \\
& =\left[\begin{array}{ccc}
0 & (-1)^{L} & 0 \\
(-1)^{L} & 0 & (-1)^{L-1}(K+L-1) \\
0 & 0 & \overline{1}
\end{array}\right],
\end{aligned}
$$

while for the inverse transformation the matrix is $\nabla^{-1}=\Xi_{L}^{-1} \nabla$. The overall transformation from the orthohexagonal $C_{1}$ setting is obtained through the matrices ${ }^{\nabla} \Gamma_{L}=\Pi \nabla \Xi_{L}$ and the correspondent inverse ones.

\subsection{Axial settings for $3 K$-layer polytypes}

The length of $c_{n}$ for Subclass $L$ in the fixed-angle setting can be calculated from (4), in which $p=(-1)^{L} 2(K+L-1)$ and $q=0$ (Class $\left.a\right)$ or $p=0$ and $q=(-1)^{L} 2(K+L-1)($ Class $b)$. The result is $(a$
Table 3. Basic and Fixed-angle axial settings for $N$-layer polytypes

Number of layers

$3 K+1 \quad 3^{0}(\bmod 3)$

$3 K+2 \quad 2 \times 3^{0}(\bmod 3)$

$9 K+3 \quad 3^{1}\left(\bmod 3^{2}\right)$

$9 K+6 \quad 2 \times 3^{1}\left(\bmod 3^{2}\right)$

$27 K+9 \quad 3^{2}\left(\bmod 3^{3}\right)$

$27 K+18 \quad 2 \times 3^{2}\left(\bmod 3^{3}\right)$.

$81 K+27 \quad 3^{3}\left(\bmod 3^{4}\right)$

$81 K+542 \times 3^{3}\left(\bmod 3^{4}\right)$

Basic axial
setting
${ }^{(a ; b)} \mathbf{S}_{1}$
${ }^{(a ; b)} \mathbf{S}_{1}$
${ }^{(a ; b)} \mathbf{S}_{3}$
${ }^{(a ; b)} \mathbf{S}_{3}$
${ }^{(a ; b)} \mathbf{S}_{9}$
${ }^{(a ; b)} \mathbf{S}_{9}$
${ }^{(a ; b)} \mathbf{S}_{27}$
${ }^{(a ; b)} \mathbf{S}_{27}$

$c_{n}$

$-$

$+$

$-$

$+$

$-$

$+$

$-$ or $b$ according to the Class):

$$
c_{n}=(-K-L / 3)(a, b)=-(3 K+L)(a, b) / 3,
$$

where $a$ and $b$ are the lengths of the axes of the spacefixed setting. The projection $c_{n}$ is thus translationally equivalent to $\pm 1 / 3$ along $a$ or $b$, where minus and plus signs are respectively for Subclass 1 and 2 . For $N=3 K$, the Fixed-angle setting cannot be used [equation (9)]; in fact it would have $c_{n}=-K(a, b)$, translationally equivalent to $(0,0)$. This value is not possible for the non-orthogonal polytypes. However (Fig. 3), the Standard setting of a polytype with $3^{n}$ layers $\left(3^{n}, a \mathbf{S}\right.$ and $\left.{ }^{3^{n}, b} \mathbf{S}\right)$ can be used as basic axial setting for polytypes with a number of layers that is a multiple of $3^{n}$, but different from $3^{n+m}$ or multiple $(m>0)$, exactly in the same way as the 1-layer based setting can be used as basic axial setting for Class a polytypes with $N \neq 3$. The resulting fixed-angle settings are labelled ${ }^{3^{n}, a} \mathbf{F}$ and ${ }^{3^{n}, b} \mathbf{F}$, respectively for Class $a$ and Class $b$. In this way, the number of

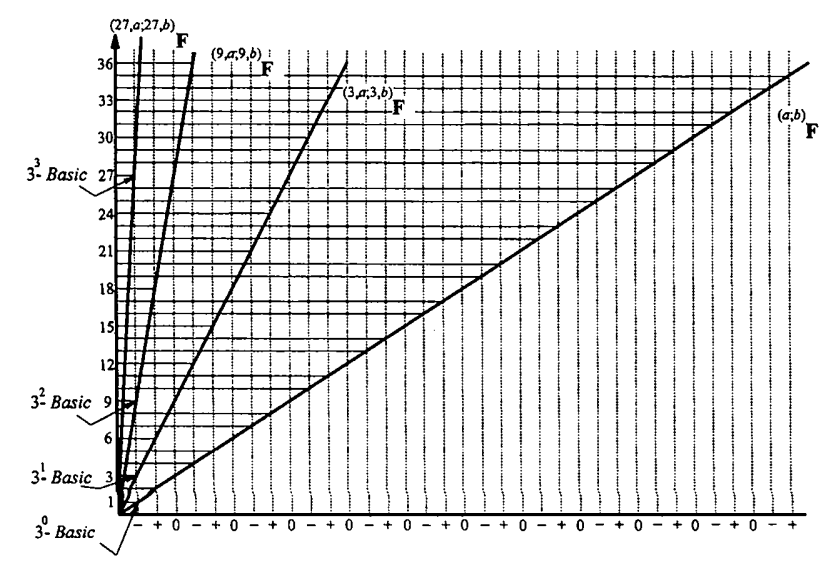

Fig. 3. Definition of Fixed-angle setting for $N$-layer polytypes. Ordinate: the number of layers. Abscissa: the component of $\mathbf{c}_{n}$ (along $a$ or $b$ of the space-fixed reference, depending on the Class), expressed as,-+ and 0 . The thick lines represent the $c$ axes of the Fixed-angle settings indicated by the corresponding labels. The intersection of a line parallel to the abscissa with $c$ gives the reduced component of $\mathbf{c}_{n}$. When this value is zero, the corresponding basic axial setting cannot be used to build a Fixedangle setting. Note especially settings of polytypes with $N=0(\bmod 3)$ 
Table 4. Example of polytype classification according to K, Series and Subclass

\begin{tabular}{|c|c|c|c|c|c|c|}
\hline$K$ & Subclass & Number of layers & Number of layers & $\begin{array}{c}\text { Series } 2 \\
\text { Number of layers }\end{array}$ & $\begin{array}{c}\text { Series } 3 \\
\text { Number of layers }\end{array}$ & $\begin{array}{c}\mathbf{S} \rightarrow \mathbf{F} \\
\underset{\text { Transformation }}{\text { matrix }}\end{array}$ \\
\hline 0 & 2 & 2 & 6 & 18 & 54 & $\Xi_{2}$ \\
\hline 1 & 1 & 4 & 12 & 36 & 108 & $\Xi_{1}$ \\
\hline 1 & 2 & 5 & 15 & 45 & 135 & $\Xi_{2}$ \\
\hline 3 & 1 & 10 & 30 & 90 & 270 & $\Xi_{1}$ \\
\hline 3 & 2 & 11 & 33 & 99 & 297 & $\Xi_{2}$ \\
\hline 4 & 1 & 13 & 39 & 117 & 351 & $\Xi_{1}$ \\
\hline 4 & 2 & 14 & 42 & 126 & 378 & $\Xi_{2}$ \\
\hline
\end{tabular}

Table 5. Cell parameters in different axial setting for two polytypes (example)

The parameters have been calculated assuming ideal parameters for the $1 M$ polytype as $a=5.2, b=9.0, c=10 \AA$, $\beta=100^{\circ}$. Projection $c_{n}$ is given making reference to the $a$ and $b$ axes of $C_{1}$ setting.

\begin{tabular}{|c|c|c|c|c|c|c|c|c|c|c|c|c|}
\hline Polytype & Setting & $c_{n}$ & $a(\AA)$ & $b(\AA)$ & $c(\AA)$ & $\alpha\left(^{\circ}\right)$ & $\beta\left({ }^{\circ}\right)$ & $a^{*}\left(\AA^{-1}\right)$ & $b^{*}\left(\AA^{-1}\right)$ & $c^{*}\left(\AA^{-1}\right)$ & $\alpha^{*}\left({ }^{\circ}\right)$ & $\beta^{*}\left({ }^{\circ}\right)$ \\
\hline 8 layers, & $C_{1}$ & $(0,0)$ & 5.2 & 9.0 & 236.4 & 90 & 90 & 0.192 & 0.111 & 0.00423 & 90 & 90 \\
\hline \multirow[t]{2}{*}{ Class $a$} & ${ }^{a} \mathbf{S}$ & $(\overline{\overline{1}} / 3,0)$ & 5.2 & 9.0 & 78.8 & 90 & 91.3 & 0.192 & 0.111 & 0.0127 & 90 & 88.7 \\
\hline & ${ }^{a} \mathbf{F}$ & $(8 / 3,0)$ & 5.2 & 9.0 & 80.0 & 90 & 100 & 0.195 & 0.111 & 0.0127 & 90 & 80 \\
\hline 11 layers, & $C_{1}$ & $(0,0)$ & 5.2 & 9.0 & 325.1 & 90 & 90 & 0.192 & 0.111 & 0.00308 & 90 & 90 \\
\hline \multirow[t]{3}{*}{ Class $b$} & ${ }^{b} \mathbf{T}$ & $(0, \overline{1} / 3)$ & 5.2 & 9.0 & 108.4 & 91.6 & 90 & 0.192 & 0.111 & 0.00923 & 88.4 & 90 \\
\hline & ${ }^{b} \mathbf{S}$ & $(0, \overline{1} / 3)$ & 9.0 & 5.2 & 108.4 & 90 & 91.6 & 0.111 & 0.192 & 0.00923 & 90 & 88.4 \\
\hline & ${ }^{b} \mathbf{F}$ & $(0,11 / 3)$ & 9.0 & 5.2 & 113.3 & 90 & 107.0 & 0.111 & 0.201 & 0.00923 & 90 & 73.0 \\
\hline
\end{tabular}

possible Fixed-angle settings is kept at a minimum (Table 3).

\subsection{Axial transformations}

The consequences of (3) and related definitions can be summarized as follows. The integer $n$ determines the basic axial setting common to all polytypes of the corresponding Series, while the Subclass defines the direction of the $a^{*}$ axis of the Fixed-angle setting with respect to that of the Standard setting. All polytypes belonging to a given Series can be indexed in their own Standard setting and in the Fixed-angle setting obtained by multiplying by $N / 3^{n}$ the period along $c$ of the corresponding basic axial setting. The transformation matrices between the Standard and the Fixed-angle setting depend on the Subclass $L$ and on $K$. The general transformation rule can be obtained by taking into account the relation between the monoclinic angles of polytypes in (2). For each Class, the transformation depends just on the number $N$ of layers in the polytype and on the Subclass. If $N$ represents the number of layers of a general polytype and $N^{\prime}$ the number of layers of the polytype defining the corresponding basic axial setting, by definition, $N=3^{n}(3 K+L)$ and $N^{\prime}=3^{n}$ hold. Therefore, the ratio

$$
N / N^{\prime}=3^{n}(3 K+L) / 3^{n}=3 K+L
$$

is completely independent of the Series. Polytypes belonging to the same Subclass and with the same value of $K$ transform from the Standard setting to the respective Fixed-angle setting exactly in the same way, by means of the same matrix (see Table 4). This result is also independent of the Class (if one starts from the standard, and not the transitional setting of Class b), since the special features defining a Class are contained in the axial parameters and not in the transformation rules. An example of classification is given in Table 4. Cell parameters in the above introduced settings are shown in Table 5 for an 8-layer Class $a$ polytype and an 11-layer Class $b$ polytype.

\section{Discussion and conclusions}

Mica polytypes can be divided into orthogonal and nonorthogonal groups. In the case of orthogonal polytypes, the standard $\left({ }^{(a, b)} \mathbf{S}\right)$ and fixed-angle $\left(\left(^{\left(3^{n}, a ; 3^{n}, b\right)} \mathbf{F}\right)\right.$ settings coincide; therefore, $c$ coincides with $c_{o}, \mathbf{c}_{n}=(0,0)$ and the choice of the axial setting is simple. However, in the case of orthogonal polytypes and of Class $b$ polytypes, additional checks on the space orientation have to be considered, since there are respectively six and three orientations leading to the same projection $\mathbf{c}_{n}$ (Zvyagin, 1967). This additional check is accomplished by taking into account the symmetry transformation rules among 
Table 6. Features of the axial settings for $N$-layer mica polytypes

$\begin{array}{ccccc}a\left({ }^{a} \mathbf{S}\right)=a\left({ }^{b} \mathbf{T}\right)=a\left({ }^{a} \mathbf{F}\right)=a\left(C_{1}\right) ; & a\left({ }^{b} \mathbf{S}\right)=a\left({ }^{b} \mathbf{F}\right)=b\left(C_{1}\right) ; & b\left({ }^{a} \mathbf{S}\right)=b\left({ }^{b} \mathbf{T}\right)=b\left({ }^{a} \mathbf{F}\right)=b\left(C_{1}\right) ; & b\left({ }^{b} \mathbf{S}\right)=b\left({ }^{b} \mathbf{F}\right)=a\left(C_{1}\right) . \\ \text { Orthogonal setting } \dagger & { }^{a} \mathbf{S} \text { setting } /{ }^{b} \mathbf{S} \text { setting } & { }^{b} \mathbf{T} \text { setting } & { }^{a} \mathbf{F} \text { setting } & { }^{b} \mathbf{F} \text { setting } \\ \mathbf{c}_{n}=(0,0) & \mathbf{c}_{n}=(-1 / 3,0) & \mathbf{c}_{n} n=(0,-1 / 3) & \mathbf{c}_{n}(N)=(-N / 3,0) & \mathbf{c}_{n}(N)=(-N / 3,0) \\ c(N) / c\left(N^{\prime}\right)=N / N^{\prime} & c(N) / c\left(N^{\prime}\right)=N \sin \beta_{N} / N^{\prime} \sin \beta_{N^{\prime}} & c(N) / c\left(N^{\prime}\right)=N \sin \alpha_{N} / N^{\prime} \sin \alpha_{N^{\prime}} & c(N) / c\left(N^{\prime}\right)=N / N^{\prime} & c(N) / c\left(N^{\prime}\right)=N / N^{\prime} \\ \alpha=\beta=90^{\circ} & \alpha_{N}=90^{\circ} & \tan \alpha_{N}=-3 c_{o}(N) / b & \alpha_{N}=90^{\circ} & \alpha_{N}=90^{\circ} \\ & \tan \beta_{N}=-3 c_{o}(N) / a & \beta_{N}=90^{\circ} & \beta_{N}=\beta_{1 M} & \tan \beta_{N}=\frac{1}{2} \tan \beta_{2 M_{2}}\end{array}$

$\uparrow$ Based on orthohexagonal axes.

$Z$ symbols (Zvyagin, 1974). In the case of non-orthogonal polytypes, the choice of the axial setting is of critical importance, especially when the number of layers increases. The initial transformation from the $C_{1}$ setting is accomplished by matrix $\Pi$, leading to ${ }^{a} \mathbf{S}$ [Class $a$ : equation (1)] or ${ }^{b} \mathbf{T}[$ Class $b$ : equation (1a)] settings; $a$ and $b$ axes of these two settings always coincide with those of the space-fixed reference. In the other settings, these axes can have the same or opposite orientation with respect to the space-fixed reference, depending on the number of layers, but the Fixed-angle setting for both Classes has the same monoclinic angle as the basic axial setting. The general features are summarized in Table 6. In all cases, for two general polytypes with $N$ and $N^{\prime}$ layers, $c_{o}(N) / c_{o}\left(N^{\prime}\right)=N / N^{\prime}$. Therefore, in the ${ }^{(a, b)} \mathrm{S}$ settings, the length $c_{n}$ is fixed and the monoclinic angle changes with the number of layers. The opposite is true in the ${ }^{\left(3^{n}, a ; 3^{n}, b\right)} \mathbf{F}$ settings.

The ${ }^{(a, b)} \mathbf{S}$ settings correspond to the conventional cell and to a projection $c_{n}(\overline{1} / 3,0)$, i.e. the minimal value for all non-orthogonal polytypes. These settings have thus often been chosen when describing micas (see, for example, Hendricks \& Jefferson, 1939; Peacock \& Ferguson, 1943; Smith \& Yoder, 1956). However, the $\left(3^{n}, a ; 3^{n}, b\right)$ F settings can be singled out using just the Class and the number of layers, and the transformations towards the other possible settings are straightforward. For this reason, they have some advantages over ${ }^{(a, b)} \mathbf{S}$, especially when dealing with the reciprocal lattice.

Let the period along $c^{*}$ for $1 M$ polytype be $c_{1}^{*}$ (it is about $0.1 \mathrm{~A}^{-1}$ ). The diffraction pattern of an $N$-layer mica polytype shows $N$ spots along any reciprocallattice row parallel to $c^{*}$ within the $c_{1}^{*}$ repeat. Some of these spots are ideally absent: they correspond to the additional reflection conditions (as defined in Hahn \& Vos, 1983), which can be interpreted in terms of noncharacteristic crystallographic orbits (Engel et al., 1984) and by means of the OD theory (Dornberger-Schiff, 1966). However, especially in the case of dioctahedral micas (Takeda \& Ross, 1995), where the deviations from the ideal geometry are larger, the additional reflection conditions are violated and the corresponding spots can appear, although with a weak intensity. Because of the large number of reflections, the choice of the correct axial setting is not straightforward, at least with two-dimensional patterns containing the $c^{*}$ axis. In this case, since, for each Class and $N,\left(^{\left(3^{n}, a ; 3^{n}, b\right)} \mathbf{F}\right.$ settings are known a priori, they are suggested to be, at least initially, used.

\subsection{Applications}

5.1.1. $4 M_{3}$ polytype. As an example of application of the diffraction pattern interpretation based on the settings presented in this paper, the case of the $4 M_{3}$ polytype, belonging to Class a, Subclass 1, and described by $Z$ symbols 3531 and RTW symbols $2 \overline{2} 2 \overline{2} \uparrow$ is discussed. This polytype was identified by means of oblique-texture electron diffraction (Zhukhlistov et al., 1990). These authors made reference to the orthohexagonal setting, having a repeat of 12 layers along the $c$ axis, in which the reflection conditions are given in the synthetic expression

$$
k=3 n: l_{12}=12 n \pm 4 ; \quad k \neq 3 n: l_{12} \neq 4 n+2,
$$

where $l_{12}$ means that the $l$ index is referred to the 12 layer orthohexagonal unit cell. These synthetic reflection conditions have to be interpreted by taking into account the condition of integrality of monoclinic $l$ indices $\left(l_{a \mathrm{~S}}\right)$; then, they match the reflection conditions calculated (in the same cell) by means of Zvyagin's functions (Zvyagin, 1967) and shown in the $C_{1}$ row of Table 7. By applying matrix $\Pi$ [equation (1)], the transformation towards ${ }^{a} \mathbf{S}$ setting implies ( $h$ and $k$ unchanged)

$$
l_{C_{1}}=h_{a \mathbf{S}}+3 l_{a \mathbf{s}} ; \quad l_{a \mathbf{S}}=\left(-h_{C_{1}}+l_{C_{1}}\right) / 3 .
$$

$\dagger$ Zvyagin's orientation symbols ( $Z$ symbols) give the space-fixed intralayer and interlayer displacements in phyllosilicates as a sequence of six digits (1-6) and three symbols $(0,+,-)$, respectively (Zvyagin et al., 1979). However, in the case of micas built by centrosymmetric layers, shortened symbols giving the space-fixed orientation of corresponding layers in a polytype have been introduced (Zhukhlistov et al., 1990): they are written as a sequence of $N$ digits 1-6. RTW orientation-free symbols (Ross et al., 1966) are written as a sequence of $N$ digits $0, \pm 1, \pm 2,3$, the $j$ th symbol giving the rotation angle between $j$ th and $(j+1)$ th layers as integer multiple of $60^{\circ}$. They can be obtained as the difference between pairs of $Z$ symbols. It is now common practice to include RTW symbols inside square brackets. However, this was not stated by the authors in their original paper (Ross et al., 1966). 
Table 7. Distinctive lattice parameters and reflection conditions for $4 M_{3}$ polytype $(Z=3531 ; R T W=2 \overline{2} \overline{2})\left(c_{n}\right.$ is referred to $a$ and $b$ axes of $C_{1}$ setting)

$a$ and $b$ are the same as for the $1 M$ polytype in all settings. In the ${ }^{a} \mathbf{F}$ setting, the reflection conditions assume the simplest expression.

$\begin{array}{lcccc}\text { Setting } & c_{n} & \beta\left(^{\circ}\right) & c(\AA) & a^{*}\left(\AA^{-1}\right) \\ C_{1} & (0,0) & 90 & 157.57 & 0.192 \\ { }^{a} \mathbf{S} & (\overline{1} / 3,0) & 92.5 & 39.43 & 0.192 \\ { }^{a} \mathbf{F} & (\overline{4} / 3,0) & 100 & 40.00 & 0.195\end{array}$

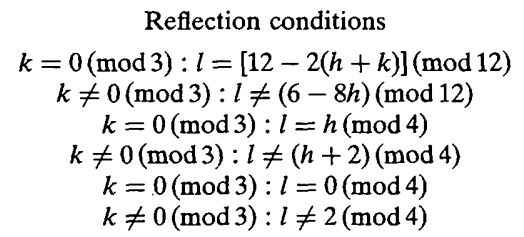

Taking into account the $C$-centring condition $h+k=0(\bmod 2), l_{a \mathrm{~S}}$ takes the following values $(m$ integer):

$$
\begin{aligned}
& k=0(\bmod 3), k \text { even }: h=2 m, k=6 m ; \\
& l_{a_{\mathbf{S}}}=4-6 m(\bmod 4)=4-3 h(\bmod 4)=h(\bmod 4) \\
& k=0(\bmod 3), k \text { odd }: h=2 m+1, k=6 m+3 ; \\
& l_{a_{\mathbf{S}}}=4-3-6 m(\bmod 4)=4-3 h(\bmod 4) \\
& \quad=h(\bmod 4) \\
& k \neq 0(\bmod 3): l_{a_{\mathbf{S}}} \neq 2-3 h(\bmod 4) .
\end{aligned}
$$

Substituting the four possible $h(\bmod 4)$ values in the last equation, a simpler expression is obtained:

$$
k \neq 0(\bmod 3): l_{a \mathbf{S}} \neq(h+2)(\bmod 4)
$$

and the complete expression is as in the ${ }^{a} \mathbf{S}$ row of Table 7. Since this example belongs to Subclass 1 and corresponds to $K=1$ [equation (3)], the transformation into the ${ }^{a} \mathbf{F}$ setting is accomplished by matrix $\Xi_{1}(K=1)$ if starting from ${ }^{a} \mathbf{S}$ setting, or by matrix $\Gamma_{1}(K=1)$, if starting from $C_{1}$ setting. Indices $h$ and $k$ remain again unchanged while the relation between $l_{a_{S}}\left(l_{C_{1}}\right)$ and $l_{a_{F}}$ is

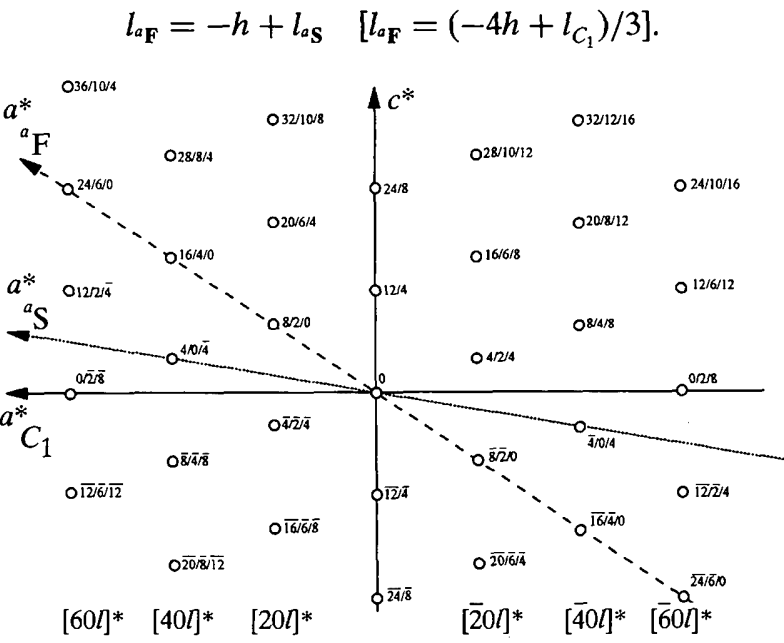

Fig. 4. ( $h 0 l)$ reciprocal-lattice plane of $4 M_{3}(Z=3531$; RTW $=2 \overline{2} \overline{2} 2)$ mica lattice. The scale along $c^{*}$ and $a^{*}$ axes is not the same. Indexing: the three numbers represent the $l$ index according to $C_{1}$, ${ }^{a} \mathbf{S}$ and ${ }^{a} \mathbf{F}$, respectively. For $00 l$, indexing according to both ${ }^{a} \mathbf{S}$ and ${ }^{a} \mathbf{F}$ coincides.
The complete expression of the reflection conditions in the ${ }^{a} \mathbf{F}$ setting takes the simplest expression as can be seen in the ${ }^{a} \mathbf{F}$ row of Table 7 . The axial settings for $(h 0 l)$ and $(h h l)$ planes are shown in Figs. 4 and 5, respectively: the axes of the $C_{1}$ setting are fixed, while those of the other two settings can be easily found on the basis of Table 7. Some more examples, including a detailed analysis of the geometrical features of the patterns of 2-layer polytypes $\left(2 M_{1}\right.$ and $\left.2 M_{2}\right)$ are given in Nespolo et al. (1997).

5.1.2. The PID function. The most powerful tool to determine the stacking sequences of mica polytypes from diffraction patterns is the periodic intensity distribution (PID) function $S^{N}$ (Takeda, 1967; Takeda \& Sadanaga, 1969; Takeda \& Ross, 1995), which represents the Fourier transform of the stacking sequence. The PID is obtained by removing the effect of intensity modulation by the Fourier transform of the single layer from the structure factor. As shown by Takeda \& Ross (1995), who adopted the TS unit layers (Takeda \& Sadanaga, 1969), the Fourier transform of the stacking sequence can be computed with only the displacements of the TS unit layers in the plane of the layer and without any rotational operation with respect to a given axial setting. For the most common polytypes (those

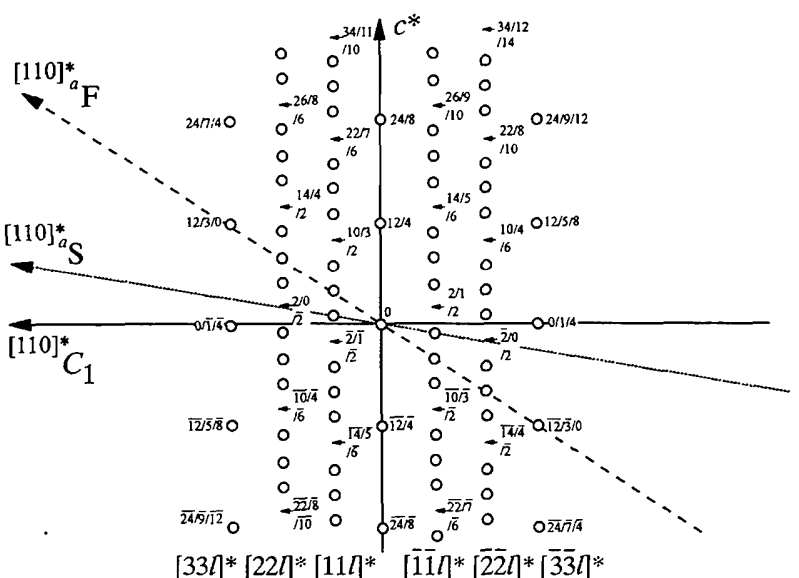

Fig. 5. $(h h l)$ plane of $4 M_{3}$ mica lattice. For explanations, see Fig. 4. Along reciprocal-lattice rows, $h= \pm 3$ nodes corresponding to nonextinguished reflections (one out of four) are indexed, while along the other rows nodes corresponding to extinguished reflections (one out of four) are indexed. 
described only by one of the four TS layers), the $c$ axis passing through the origin of each layer is displaced $-a / 3$ (Subclass 1) or $+a / 3$ (Subclass 2) for each stacking of layers (Takeda \& Ross, 1995). The corresponding axial setting is just the Fixed-angle one. The definition of ${ }^{\left(3^{n}, a ; 3^{n}, b\right)} \mathbf{F}$ settings presented in this paper includes and generalizes the criterion used by Takeda \& Ross (1995) to all possible polytypes, such as given in their Tables 4, 7, 8 and 10 .

Without a common setting, comparisons of the observed and calculated PID in principle have to be repeated $2 N$ times, by shifting of the origin and inversion of the sequence. With the ${ }^{\left(3^{n}, a ; 3^{n}, b\right)} \mathbf{F}$ settings, the symmetry of the PID function can be expressed with simpler reflection conditions.

We wish to thank Professor Boris B. Zvyagin (IGEM, Moscow) for useful criticism and suggestions and for critical reading of an earlier version of the manuscript; Professor Svetlana Soboleva (IGEM, Moscow), Professor Yoshio Takéuchi (Nihon University, Tokyo) and Professor Jill Banfield (University of WisconsinMadison) for critical reading of the manuscript. Professor Ryoichi Sadanaga (Japan Academy, Tokyo), Professor Takeo Matsumoto (University of Kanazawa) and Dr Akiji Yamamoto (NIRIM, Tsukuba) gave us the opportunity of profitable discussions. Comments and suggestions from Professor Slavomil Durovič (Slovak Academy of Sciences, Bratislava) and from an anonymous referee contributed to improve the clearness of the manuscript. One of us (MN) is indebted to Professor Masamichi Miyamoto (University of Tokyo) for his constant encouragement during this work. This research has been developed as part of a Doctoral Program (MN) supported by the Japanese Ministry of Education, Science and Culture. Financial support from the Italian Research Council (CNR) is acknowledged (GF).

\section{References}

Arnold, H. (1983). International Tables for Crystallography, Vol. A, edited by Th. Hahn, Section 5. Dordrecht/Boston: Kluwer.

Backhaus, K.-O. \& Ďurovič, S. (1984). Clays Clay Miner. 32, 453-463.

Brown, B. E. (1978). Am. Mineral. 63, 332-336.

Burzlaff, H., Zimmermann, H. \& de Wolff, P. M. (1983). International Tables for Crystallography, Vol. A, edited by Th. Hahn, Section 9.1. Dordrecht/Boston: Kluwer.

Dekeyser, W. \& Amelinckx, S. (1953). Comptes Rendus de la XIX Session Congrès Geologique International. Comité International pour l'Etude des Argiles, Algérie. Fascicule XVIII.

Dornberger-Schiff, K. (1959). Acta Cryst. 12, 173.

Dornberger-Schiff, K. (1966). Acta Cryst. 21, 311-322.
Dornberger-Schiff, K., Backhaus, K.-O. \& Ďurovič, S. (1982). Clays Clay Miner. 30, 364-374.

Dornberger-Schiff, K., Ďurovič, S. \& Zvyagin, B. B. (1982). Cryst. Res. Technol. 17, 1449-1457.

Durovič, S., Weiss, Z. \& Backhaus, K.-O. (1984). Clays Clay Miner. 32, 464-474.

Engel, P., Matsumoto, T., Steinamann, G. \& Wondratschek, H. (1984). Z. Kristallogr. Suppl. Issue No. 1.

Guinier, A., Bokij, G. B., Boll-Dornberger, K., Cowley, J. M., Ďrovič, S., Jagodzinski, H., Khrisna, P., de Wolff, P. M., Zvyagin, B. B., Cox, D. E., Goodman, P., Hahn, Th., Kuchitsu, K. \& Abrahams, S. C. (1984). Acta Cryst. A40, 399-404.

Hahn, Th. (1983). International Tables for Crystallography, Vol. A, edited by Th. Hahn, Section 1.1. Dordrecht/Boston: Kluwer.

Hahn, Th. \& Vos, A. (1983). International Tables for Crystallography, Vol. A, edited by Th. Hahn, Section 2.13 . Dordrecht/Boston: Kluwer.

Hendricks, S. B. \& Jefferson, M. E. (1939). Am. Mineral. 24, 729-771.

Merlino, S. (1990). Period. Mineral. 59, 69-92.

Nespolo, M., Takeda, H. \& Ferraris, G. (1997). Modular Aspects of Minerals. EMU Notes in Mineralogy, Vol. 1, edited by S. Merlino, pp. 81-118. Budapest: Eötvös University Press.

Pabst, A. (1955). Am. Mineral. 40, 967-974.

Peacock, M. A. \& Ferguson, R. B. (1943). Univ. Toronto Stud. Miner. 48, 65-82.

Radoslovich, E. W. (1959). Nature (London), 183, 253.

Radoslovich, E. W. \& Norrish, K. (1962). Am. Mineral. 47, 599-616.

Ross, M., Takeda, H. \& Wones, D. R. (1966). Science, 151, 191-193.

Sadanaga, R. \& Takeda, H. (1968). Acta Cryst. B24, 144-149.

Smith, J. V. \& Yoder, H. S. (1956). Mineral. Mag. 31, 209-235.

Takeda, H. (1967). Acta Cryst. 22, 845-853.

Takeda, H. \& Ross, M. (1995). Am. Mineral. 80, 715-724.

Takeda, H. \& Sadanaga, R. (1969). Mineral. J. 5, 434-449.

Takéuchi, Y. \& Haga, N. (1971). Mineral. Soc. Jpn Spec. Pap. 1, 74-87. (Proc. IMA-IAGOD Meetings '70, IMA Vol.)

Thompson, J. B. Jr (1981). Structure and Bonding, edited by M. O'Keefe \& A. Navrotsky, Vol. 2, pp. 167-196. New York/ London/Toronto/Sydney/San Francisco: Academic Press.

Weiss, Z. \& Wiewióra, A. (1986). Clays Clay Miner. 34, 53-68.

Zhukhlistov, A. P., Zvyagin, B. B. \& Pavlishin, V. I. (1990). Sov. Phys. Crystallogr. 35, 232-236.

Zvyagin, B. B. (1962). Sov. Phys. Crystallogr. 6, 571-580.

Zvyagin, B. B. (1967). Electron Diffraction Analysis of Clay Mineral Structures. New York: Plenum Press.

Zvyagin, B. B. (1974). 2nd European Crystallographic Meeting, Keszthely, Collected Abstracts, pp. 162-164.

Zvyagin, B. B. (1997). Modular Aspects of Minerals. EMU Notes in Mineralogy, Vol. 1, edited by S. Merlino, pp. 345-372. Budapest: Eötvös University Press.

Zvyagin, B. B. \& Drits, V. A. (1996). Clays Clay Miner. 44, 297-303.

Zvyagin, B. B., Vrublevskaya, Z. V., Zhukhlistov, A. P., Sidorenko, O. V., Soboleva, S. V. \& Fedotov, A. F. (1979). High-Voltage Electron Diffraction in the Study of Layered Minerals. Moscow: Nauka. (In Russian.) 